\title{
Planetary nebulae detected in the Spitzer Space Telescope GLIMPSE 3D Legacy Survey
}

\author{
Yong Zhang ${ }^{1}$, Sun Kwok ${ }^{1}$, Chih-Hao Hsia ${ }^{1}$, Jun-ichi Nakashima ${ }^{1}$ \\ and Nico Koning ${ }^{2}$ \\ ${ }^{1}$ Department of Physics, The University of Hong Kong, Hong Kong, China \\ email: zhangy96@hku.hk \\ ${ }^{2}$ Department of Physics and Astronomy, University of Calgary, Calgary, Canada T2N 1N4
}

\begin{abstract}
Due to interstellar extinction, optical census of Galactic planetary nebulae (PNs) is highly incomplete, and some compact $\mathrm{H}$ II regions might have been mis-classified as PNs. The problem is particularly severe in the Galactic plane where the extinction in the optical is significant and hampers the detections of PNs. Unlike optical observations, infrared (IR) observations are hardly affected by interstellar extinction, and provide a good opportunity to study highly obscured PNs. In this study, we use the data from the Spitzer Legacy Infrared Mid-Plane Survey Extraordinaire 3D (GLIMPSE 3D) to investigate the mid-infrared (MIR) properties of PNs and PN candidates.
\end{abstract}

Keywords. infrared: ISM, planetary nebulae: general, planetary nebulae: individual (M 1-41), stars: AGB and post-AGB

\section{Introduction}

As of 2011, approximately 3000 planetary nebulae (PNs) have been discovered in the Galaxy through various optical surveys. However, the number of known Galactic PNs is still about one order of magnitude smaller than the theoretical prediction. One of the major reasons for this is the substantial interstellar extinction of the Galactic plane. Interstellar dust could also obscure faint extended structures of some PNs and PN candidates, leading to mis-classification of the morphology of PNs. On the other hand, PNs can emit strongly in the infrared (IR) range, where the effect of interstellar extinction is very slight. Therefore, IR observations have considerable advantages in investigating the PNs in the Galactic plane. We have been carrying out a project to study PNs using the data taken from the Spitzer Legacy Infrared Mid-Plane Survey (GLIMPSE), aiming at investigating IR properties of PNs and the spacial distribution of dust grains, detecting nebular extended structures, and searching for new PNs. Some studies based on GLIMPSE I\&II data have been presented in our previous papers (Kwok et al. 2008; Zhang \& Kwok 2009). Here we report partial results of our studies based on the GLIMPSE 3D data.

\section{Data}

The Spitzer/IRAC images, centered at approximately 3.6, 4.5, 5.8 and $8.0 \mu \mathrm{m}$, were taken from the GLIMPSE 3D dataset (Meade et al. 2007). All the four bands were simultaneously observed with a pixel resolution of about 1.2 arcsec. The GLIMPSE 3D survey extends the GLIMPSE I\&II latitude coverage to $|b|<3^{\circ}$ at nine selected strips above and below the Galactic plane, and to $|b|<4.2^{\circ}$ in the center of the Galaxy. 


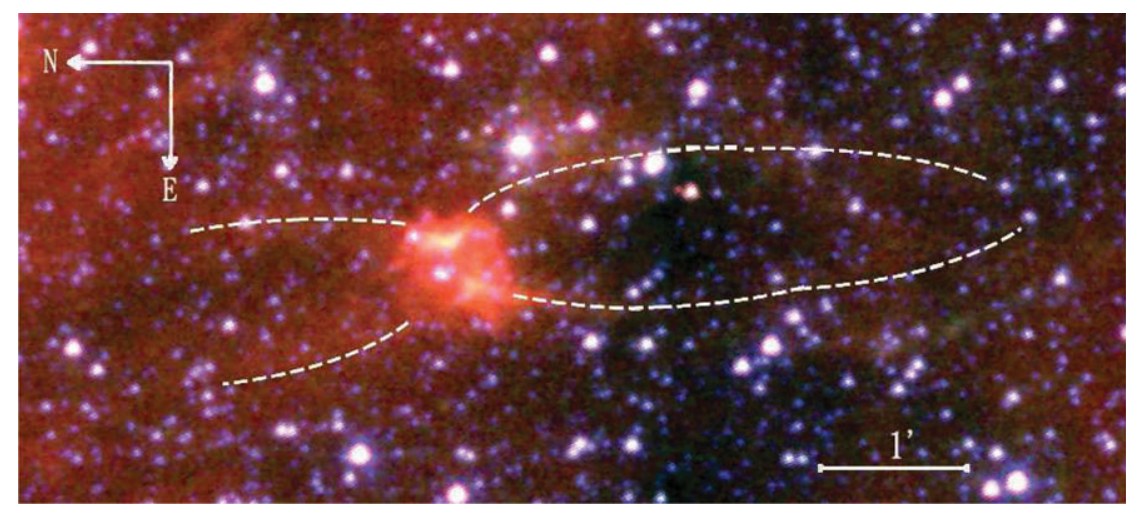

Figure 1. The IRAC image of M 1-41. The superimposed dashed lines are a sketch of the extended bipolar structures.

The total area is about $120 \mathrm{deg}^{2}$. The sample of PNs was taken from the MASH (Macquarie/AAO/Strasbourg $\mathrm{H} \alpha$ Planetary Galactic Catalogue) and MASH II catalogues (Parker et al. 2006; Miszalski et al. 2008), which contain 1000 faint, new PNs. We also searched for all the previously known PNs catalogued by Kohoutek (2001) within the GLIMPSE 3D survey area. Integrated fluxes were measured using the same approach as in our previous papers.

\section{Results}

We find that there are $228 \mathrm{MASH}$ PNs and 107 previously known PNs within the GLIMPSE 3D area, among which 90 and 101 have clearly visible IRAC counterparts. The detection rate of GLIMPSE 3D PNs is higher than that of the PNs in the GLIMPSE I\&II area, suggesting that the bright IR background emission hamper the detection of PNs in the inner Galactic plane. Most of the GLIMPSE 3D PNs are compact and redder than the field stars. Combining the fluxes with the existing photometric measurements from other IR archives, we construct spectral energy distributions, which exhibit the bound-free and photospheric emission from ionized gas and cold dust emission with a color temperature of about $100 \mathrm{~K}$. In particular, the IRAC images reveal extended bipolar structures in the PN M1-41 (Fig. 1). The southern lobe has an extension of about 3.7' from the center. The central nebulosity has an irregular appearance, and is much brighter than the lobes. Without highly sensitive imaging, it is impossible to accurately designate PN morphology.

\section{Acknowledgements}

The work was supported by a grant from the Research Grants Council of the Hong Kong Special Administrative Region, China (Project No. HKU 7031/10P) and a grant to YZ from the Seed Funding Programme for Basic Research in HKU (200909159007).

\section{References}

Kohoutek, L., 2001, A\&A, 378, 843

Kwok, S., Zhang, Y., Koning, N., Huang, H.-H., \& Churchwell, E. 2008, ApJs, 174, 426

Meade, M. R., et al. 2007, GLIMPSE3D v1.0 Data Release (23 August, 2007)

Miszalski, B., Parker, Q. A., Acker, A., Birkby, J. L., Frew, D. J., \& Kovacevic, A. 2008, MNRAS, 384,525

Parker, Q. A. et al. 2006, MNRAS, 373, 79

Zhang, Y. \& Kwok, S. 2009, ApJ, 706, 252 